\title{
THE DISCOVERY OF YOUNG, LUMINOUS, COMPACT STELLAR CLUSTERS IN THE STARBURST GALAXY NGC $253^{1}$
}

\section{ALAN M. WATSON}

Lowell Observatory, 1400 West Mars Hill Road, Flagstaff, Arizona 86001 and Department of Astronomy, New Mexico State University, Dept. 4500, Box 30001, Las Cruces, New Mexico 88003

Electronic mail: awatson@nmsu.edu

\section{JOHN S. GALLAGHER III}

Department of Astronomy, University of Wisconsin-Madison, 475 N. Charter Street, Madison, Wisconsin 53706 Electronic mail: jsg@astro.wisc.edu

\section{JON A. HOLTZMAN}

Lowell Observatory, 1400 West Mars Hill Road, Flagstaff, Arizona 86001 and Department of Astronomy, New Mexico State University, Dept. 4500, Box 30001, Las Cruces, New Mexico 88003 Electronic mail: holtz@nmsu.edu

\section{J. JEFF HESTER}

Department of Physics and Astronomy, Arizona State University, Tyler Mall, Tempe, Arizona 85287 Electronic mail: jjh@cygloop.la.asu.edu

\section{JEREMY R. MOULD}

Mount Stromlo and Siding Springs Observatories, Australian National University, Private Bag, Weston Creek Post Office, ACT 2611, Australia

Electronic mail: jrm@merlin.anu.edu.au

\section{GILDA E. BALLESTER}

Department of Atmospheric, Oceanic, and Space Sciences, University of Michigan, 2455 Hayward, Ann Arbor, Michigan 48109

\section{CHRISTOPHER J. BURROWS}

Astrophysics Division, Space Science Department, ESA and Space Telescope Science Institute, 3700 San Martin Drive, Baltimore, Maryland 21218

\section{STEFANO CASERTANO}

Astrophysics Division, Space Science Department, ESA, Space Telescope Science Institute, 3700 San Martin Drive, Baltimore, Maryland 21218 and Department of Astronomy, Johns Hopkins University, 3400 N. Charles Street, Baltimore, Maryland 21218

\section{JOHN T. CLARKE}

Department of Atmospheric, Oceanic, and Space Sciences, University of Michigan, 2455 Hayward, Ann Arbor, Michigan 48109

\section{DAVID CRISP AND ROBIN EVANS}

Jet Propulsion Laboratory, 4800 Oak Grove Drive, Pasadena, California 91109

\section{RICHARD E. GRIFFITHS}

Department of Astronomy, Johns Hopkins University, 3400 N. Charles Street, Baltimore, Maryland 21218

JOHN G. HOESSEL

Department of Astronomy, University of Wisconsin-Madison, 475 N. Charter Street, Madison, Wisconsin 53706

PAUl A. SCOWEN

Department of Physics and Astronomy, Arizona State University, Tyler Mall, Tempe, Arizona 85287

\section{KARL R. STAPELFELDT AND JOHN T. TRAUGER}

Jet Propulsion Laboratory, 4800 Oak Grove Drive, Pasadena, California 91109

JAMES A. WESTPHAL

Division of Geological and Planetary Sciences, California Institute of Technology, Pasadena, California 91125 Received 1995 December 7; accepted 1996 April 29 


\section{ABSTRACT}

We report the discovery of four young, luminous, compact stellar clusters in the central region of the starburst spiral galaxy NGC 253 . One cluster has an intrinsic $M_{V}$ of about -15 or brighter, an inferred mass in excess of $1.5 \times 10^{6} M_{\odot}$, assuming a Salpeter IMF from $0.08 M_{\odot}$ to $100 M_{\odot}$, and is resolved with a half-light radius of about 2.5 parsec if it has a radial profile similar to a Galactic globular cluster. The others have intrinsic $M_{V}$ of about -11 or brighter, inferred masses in excess of $5 \times 10^{4} M_{\odot}$, and are similarly compact. These clusters are excellent candidate young globular clusters. The brighter cluster is similar in luminosity, inferred mass, and compactness to the super star clusters found in other sites of ongoing or recent intense star formation. The location of these clusters in the midst of a starburst region suggests that intense star formation may be a key ingredient in their creation. There are hints that these candidate young globular clusters may be forming with a high specific frequency. (C) 1996 American Astronomical Society.

\section{INTRODUCTION}

In this paper we report the discovery of four young, luminous, compact clusters in the central region of NGC 253 , a nearby starburst spiral galaxy. The clusters are candidate young globular clusters.

Similar clusters have been found previously in many galaxies that are undergoing or have undergone intense star formation or starbursts. In small galaxies these clusters are known as super star clusters and have been found in NGC 1140 (Hunter et al. 1994), NGC 1569 (Arp \& Sandage 1985; Waller 1991; O'Connell et al. 1994), NGC 1705 (Melnick et al. 1985; Meurer et al. 1992; O’Connell et al. 1994), and He 2-10 (Conti \& Vacca 1994). In interacting galaxies these clusters are more commonly referred to as candidate young globular clusters and have been found in M82 (O'Connell et al. 1995), NGC 3597 (Lutz 1991; Holtzman 1995), NGC 1275 (Holtzman et al. 1992; Zepf et al. 1995), NGC 7252 (Whitmore et al. 1993; Schweizer \& Seitzer 1993), NGC 4038/4039 (Whitmore \& Schweizer 1995), and NGC 7727 (Crabtree \& Smecker-Hane 1994). Barth et al. (1995) have reported the discovery of young, luminous, compact clusters in the circumnuclear ring of NGC 6951, but the distance of this galaxy prevents especially strong limits being placed on the compactness of these clusters and there are large uncertainties in the reddening correction to the clusters. Meurer et al. (1995) have also presented a study of FOC near UV images of nine starburst galaxies, all of which contain super star clusters, but again effects of dust and age prevented them from making accurate estimates of the masses of these clusters.

NGC 253 is the most massive member of the Sculptor group (Puche et al. 1991) and is a companion to NGC 247. Its velocity field away from the central starburst region and its outer H I density contours are regular (Combes 1977; Puche 1991), and this argues strongly against a recent interaction. We adopt a distance of $2.3 \mathrm{Mpc}$ to NGC 253 (Davidge \& Pritchett 1990; Davidge et al. 1991). At this distance, 1 arcsec corresponds to 11 parsec and each pixel in PC1 corresponds to 0.5 parsec.

${ }^{1}$ Based on observations with the NASA/ESA Hubble Space Telescope, obtained at the Space Telescope Science Institute, operated by AURA, Inc. under contract to NASA.

\section{OBSERVATIONS}

We obtained observations of the nuclear region of the starburst galaxy NGC 253 with the second Wide Field and Planetary Camera (WFPC2) on the Hubble Space Telescope. WFPC2 images the center of the focal plane onto four Loral $800 \times 800$ CCDs: PC1 at a scale of about 0.046 arcsec/pixel and WF2, WF3, and WF4 at scales of about 0.1 arcsec/pixel. For more details of the instrument see Burrows (1995), Trauger et al. (1994), Holtzman et al. (1995a), and Holtzman et al. (1995b). All of our observations were taken with the CCDs cooled to $-88^{\circ} \mathrm{C}$ and have exposures above about 300 electrons in the nuclear region, so CTE effects are likely to be negligible (Holtzman et al. 1995a, Sec. 5.1).

Images though the F336W, F555W, and F814W filters (the WFPC2 wide Johnson $U$, wide Johnson $V$, and wide Kron-Cousins $I$ analogs) were taken on 1994 May 29 with the nucleus of the galaxy centered on PC1. Two exposures of 400s were taken though each filter. The F336W images contain very little signal and will not be considered further.

Images through the F656N and F675W filters (the WFPC2 narrow band $\mathrm{H} \alpha$ and wide Kron-Cousins $R$ analog) were taken on 1994 September 17 with the nucleus of the galaxy centered on WF3. We elected to use WF3 rather than PC1 to increase the signal-to-noise ratio at the expense of spatial resolution. Two exposures of 1200 s were taken through F656N and two exposures of 200s through F675W. The heliocentric velocity of NGC 253 is only $251 \mathrm{~km} \mathrm{~s}^{-1}$, so $\mathrm{H} \alpha$ is redshifted by only $5 \AA$ and remains within the bandpass of the F656N filter [see Burrows (1995) for the filter transmission curve].

We reduced the observations according to the procedure recommended by Holtzman et al. (1995a) and combined the individual images in each filter using PICCRS, an implementation of a slightly modified version of the algorithm described by Groth (1991) to combine images optimally in the presence of cosmic ray events. We adopted the synthetic natural system of Holtzman et al. (1995b) (i.e., we adopt the zero points of their Table 9 but no color terms). We denote these magnitudes $m_{555}, m_{675}$, and $m_{814}$. They are fairly close to $V, R$, and $I$ for unreddened stars (Holtzman et al. 1995b). Our adoption of this system allows us to consistently compare observed magnitudes with synthetic magnitudes derived from the transmission curves of Holtzman et al. (1995b). 


\section{THE NUCLEAR REGION}

Figure 1 (Plate 22) is a F814W image of the nuclear region with a negative logarithmic stretch over 7.5 magnitudes in surface brightness. Figure 2 (Plate 23) is a $V-I$ image with a stretch from $V-I \approx m_{555}-m_{814}=1$ (white) to $V-I \approx m_{555}-m_{814}=5$ (black). We made no attempt to smooth the F555W image to match the coarser PSF in the F814W image, so some structure does appear around very compact bright sources. Each image is about 23 arcsec to a side and is centered on the bright blob, a major feature in this region of NGC 253 (see below). The images have north 7.8 degrees clockwise from vertical and east roughly to the left. Figure 3 (Plate 24) is a false color image constructed from our PC1 images of the nuclear region in F555W and F814W. The intensity scale is roughly logarithmic and the color scale roughly linear.

Figure 4 (Plate 25) is a continuum-subtracted $\mathrm{H} \alpha$ image with a negative logarithmic stretch over 10 magnitudes in surface brightness. Subtracting the continuum precisely is difficult given the combination of the width of the F675W filter, the variations in the continuum colors, and the inclusion of strong emission lines in F675W. The image has been rotated and oversampled to correspond to the PC1 images, but has intrinsically lower resolution.

The nuclear region is very confused in the F814W image, with many compact bright and dark features. It is interesting that in the very central regions higher surface brightness is correlated with redder colors, suggesting a strong coupling between dust and stars (Witt et al. 1992; Watson 1994; Watson \& Gallagher 1996). Away from the central region to the north and west the colors are generally bluer, but the correlation between surface brightness and color remains-the dark features in the F814W image that appear to be dust clouds are actually bluer than their surroundings. It is possible that we are seeing light scattered from dust clouds that are out of the plane. To the east of the central region the colors are fairly uniform and are redder than to the north and west.

The most obvious features are the bright red compact sources at $(0.0,0.0)$ and $(-5.2,+6.5)$. These features are also present in the $J, H$, and $K$ images of Sams et al. (1994). They named the central feature the "big blob"; as we shall see, this is something of a misnomer and we shall call the feature the "bright blob." The feature to the north east corresponds ${ }^{2}$ to their "spot $a$." Most of the other features labeled by Sams et al. appear to consist of mixtures of diffuse emission and many less significant emission concentrations, with the exceptions of their spots $i$ near $(-0.5,+1.0)$ and $n$ near $(-1.0,+2.0)$, which also appear to be compact sources.

Features corresponding to the compact sources are seen in the $\mathrm{H} \alpha$ image, Fig. 4. Spot $a$ is located at the base of a streamer of $\mathrm{H} \alpha$ emission. The $\mathrm{H} \alpha$ emission confirms that a

${ }^{2}$ The labeling in Sams et al. (1994) is inconsistent between their Fig. 3 and their Fig. 5. We have adopted their Fig. 3 as truth. The correspondence in the sense of Fig. 3/Fig. 5 is: $a / m, b / j, c / k, d / n, e / l, f / i, g / g, h / b, i / c$, $j /$ missing, $k / d, l / f, m / a, 1$ arcsec WSW of $a / n$. source of ionizing radiation, presumably young stars, must be present in all of the compact sources.

Figure 5 (Plate 26) shows 1 arcsec square regions centered on the four compact sources and on a star in F555W (left with stretches that are linear in counts), F814W (center with stretches that are linear in counts), and $m_{555}-m_{814}$ (right with stretches that span 2.5 magnitudes). The bright blob is clearly resolved in the PC1 images in F555W and F814W and is split into at least two components in the F555W image. These features are not the result of cosmic ray events or hot pixels. The relative smoothness of the F814W image is real and is not simply the result of the coarser point spread function delivered by the HST at longer wavelengths. The peak moves to the west (right) from F555W to F814W. The color of the bright blob becomes redder toward the center, with the reddest pixels being more than a magnitude redder in $m_{555}-m_{814}$ than those further out. This reddening cannot be the result of a change in the point spread function with wavelength, as that would tend to make the center bluer. It seems most likely that differences between the two images are the result of patchy extinction. For this reason, attempts to "deredden" the spectrum of the bright blob are subject to large uncertainties. Spots $a, i$, and $n$ are also clearly resolved and appear to be smoother than the bright blob. Spot $n$ is clearly elongated.

The two most straightforward explanations of the compact sources are that they are young, luminous, compact stellar clusters located behind extinction or that they are holes in layers of extinction overlying regions of star formation. The latter is unlikely because all are much redder than their surroundings, indicating that they are associated with more extinction rather than less. The extinction to the compact sources must be significant to explain their very red colors, and the extinction to the bright blob must further be patchy to explain the differences between the F555W and F814W images.

\section{ASTROMETRY OF THE CLUSTERS}

The WFPC2 images enable us to measure the position of the bright blob and the other clusters with sub-arcsecond accuracy. This is vital for comparison to observations at other wavelengths, especially the $2 \mathrm{~cm}$ VLA observations of Turner \& Ho (1985) which have an angular resolution of 0.2 $\operatorname{arcsec} \times 0.1$ arcsec. The centroid of the bright blob changes slightly with wavelength, so we have chosen to measure its position in the F675W and F814W images, where the effects of dust are reduced and the bright blob has a smoother appearance.

\subsection{Astrometry using the FGS Reference}

The position of any object in the WFPC2 frames with respect to the selected pair of guide stars from the Guide Star Catalog (GSC) can be determined from the Fine Guidance Sensor (FGS) data given the location of $V 1$ axis is (93.1, 81.7 ) in WF3, the distortion properties of the WFPC2 cameras, and the orientation of the WFPC2 cameras relative to the $V 3$ axis (Cox 1994; Holtzman 1995a). The accuracy of this method can be determined from the study of the pointing 
errors reported by Burrows (1995, p. 17), who found errors as large as 1.8 arcsec with a median error of 0.86 arcsec. If the distribution of errors is Gaussian, then the $1 \sigma$ uncertainty is about 0.7 arcsec. Russell \& Williams (1986) estimate that the small scale errors in the GSC are less than about 0.4 arcsec and Russell et al. (1990) estimate that the absolute errors in the GSC are $\sigma \approx 0.5$ arcsec. Our use of the distortion correction for $\mathrm{F} 555 \mathrm{~W}$ for $\mathrm{F} 675 \mathrm{~W}$ and $\mathrm{F} 814 \mathrm{~W}$ positions results in an insignificant error of at most $0.02 \operatorname{arcsec}$ (Holtzman et al. 1995a).

Agreement between the positions of the bright blob derived for each visit was disappointing. The position derived from the May observation was 004732.832 -25 $17 \quad 19.94$ (J2000) and the position derived from the September observation was 004732.951 -25 1719.37 (J2000). The May position lies about 1.7 arcsec to the WSW of the September position. We can offer no explanation for the difference between these two positions. In particular, we have identified and checked the positions of the guide stars using the Digitized Sky Survey (DSS) and can find no obvious error. We have also established that the difference does not stem from the astrometric calibration error present in PODPS at the time of our observations (Biretta 1995).

\subsection{Astrometry using an Independent Reference}

Independent positions can be derived using two nearby foreground stars as references. One is located approximately 120 arcsec to the NE of the nucleus and is visible in WF3 in the May data. The other is located approximately 70 arcsec to the WSW of the nucleus and is visible in WF4 in the September data. Both of these stars are saturated and so their positions can only be measured with an accuracy of about a half of a WF pixel or 0.05 arcsec. We determined the positions of these stars in the wide field image of NGC 253 published by Aldering \& Bothun (1991) and kindly provided by Bothun. This image was calibrated astrometrically using about 40 stars whose positions were measured on the DSS. We determined the positions of the stars to be 004739.904 -25 1603.77 and $004728.118-251747.94$ (J2000) with an estimated $1 \sigma$ uncertainty of about 0.5 arcsec.

The FGS are located about 10 arcmin from the center of the focal plane, and so the calculated roll angle is insensitive to small positional errors. For example, a positional error of 2 arcsec would lead to a roll angle error of less than 0.2 degrees. Furthermore, we determined that the apparent position angles between features visible at both epochs differ by only about 0.06 degrees. Therefore, we were able to safely adopt the roll angles derived from the FGS references.

Using these foreground stars as references we derived the position of the bright blob to be $004732.953-251719.22$ (J2000) from the May observation and 004732.988 -25 17 19.19 (J2000) from the September observation with a $1 \sigma$ uncertainty in each determination of about 0.5 arcsec. These positions are only 0.2 and 0.5 arcsec from the positions derived from the September data using the FGS reference.
TABLE 1. Cluster astrometry.

\begin{tabular}{cccccccccc}
\hline \hline \multicolumn{8}{c}{ Offsets $(\operatorname{arcsec})$} \\
In Figures 1 to 4 & On the sky & & & & & \\
Cluster & $\Delta x$ & $\Delta y$ & $\Delta N$ & $\Delta E$ & \multicolumn{2}{c}{ J2000 } & \\
\hline Bright blob & +0.0 & +0.0 & +0.00 & +0.00 & 0047 & 32.964 & -25 & 17 & 19.26 \\
Spot $a$ & -5.2 & +6.5 & +5.66 & +6.04 & 0047 & 33.410 & -25 & 17 & 13.60 \\
Spot $i$ & -0.5 & +1.2 & +1.14 & +0.66 & 0047 & 33.013 & -25 & 17 & 18.12 \\
Spot $n$ & -1.0 & +2.2 & +2.06 & +1.35 & 0047 & 33.063 & -25 & 17 & 17.20 \\
TH2 & -2.6 & +2.5 & +2.13 & +2.91 & 0047 & 33.179 & -25 & 17 & 17.13 \\
\hline \hline
\end{tabular}

\subsection{Our Adopted Positions}

We took the position of the bright blob to be the average of our three consistent positions: those derived from the May and September observations using the nearby stars as references and the September observations using the FGS reference. This average position is $004732.964-2517 \quad 19.26$ (J2000) with a $1 \sigma$ uncertainty of about 0.3 arcsec. The offsets to the bright blob from the NE and WSW stars can be determined from the information above to an accuracy of better than 0.1 arcsec. We converted these $\mathrm{J} 2000$ coordinates to the B1950 system used by previous observers using routines written by J. Bennett and incorporated into the NASA Extragalactic Database. The average position of the bright blob is $004505.580-253341.08$ (B1950). The offsets and positions of all the clusters are given in Table 1 .

\section{OBSERVED PROPERTIES OF THE CLUSTERS}

In this section, we shall present those properties of the bright blob and the other clusters that can be derived without recourse to stellar population models.

\subsection{Location}

Turner \& Ho (1985) suggest that their source TH 2, the central core of the $2 \mathrm{~cm}$ emission, is the nucleus of NGC 253. We show the astrometry of TH 2 in Table 1 along with the clusters. TH 2 is $3.6 \operatorname{arcsec}(11 \sigma)$ to the NE of our adopted position for the bright blob. We confirm and significantly strengthen the conclusions of Piña et al. (1992) and Sams et al. (1994) that the bright blob is not coincident with the radio core. Adopting our average position for the bright blob places the radio core near $(-2.6,+2.5)$ in Figs. $1-4$ and slightly to the NW of the region labeled spot $g$ by Sams et al. (1994).

If we assume that the clusters lie in the plane of the galaxy and adopt the line of nodes (49 degrees) and inclination (72 degrees) found by Puche et al. (1991), we find that the clusters lie between 35 and 55 parsec from the radio core. It is conceivable that the clusters lie in a circumnuclear ring, although such a ring would be smaller than the kiloparsecsized rings more typically found in strongly barred galaxies.

\subsection{Size}

The compactness of a star cluster is commonly characterized by a core radius $R_{\text {core }}$ (the radius at which the surface brightness drops to half of the peak surface brightness) and a half-light, effective, or median radius $R_{0.5}$ (the radius enclos- 

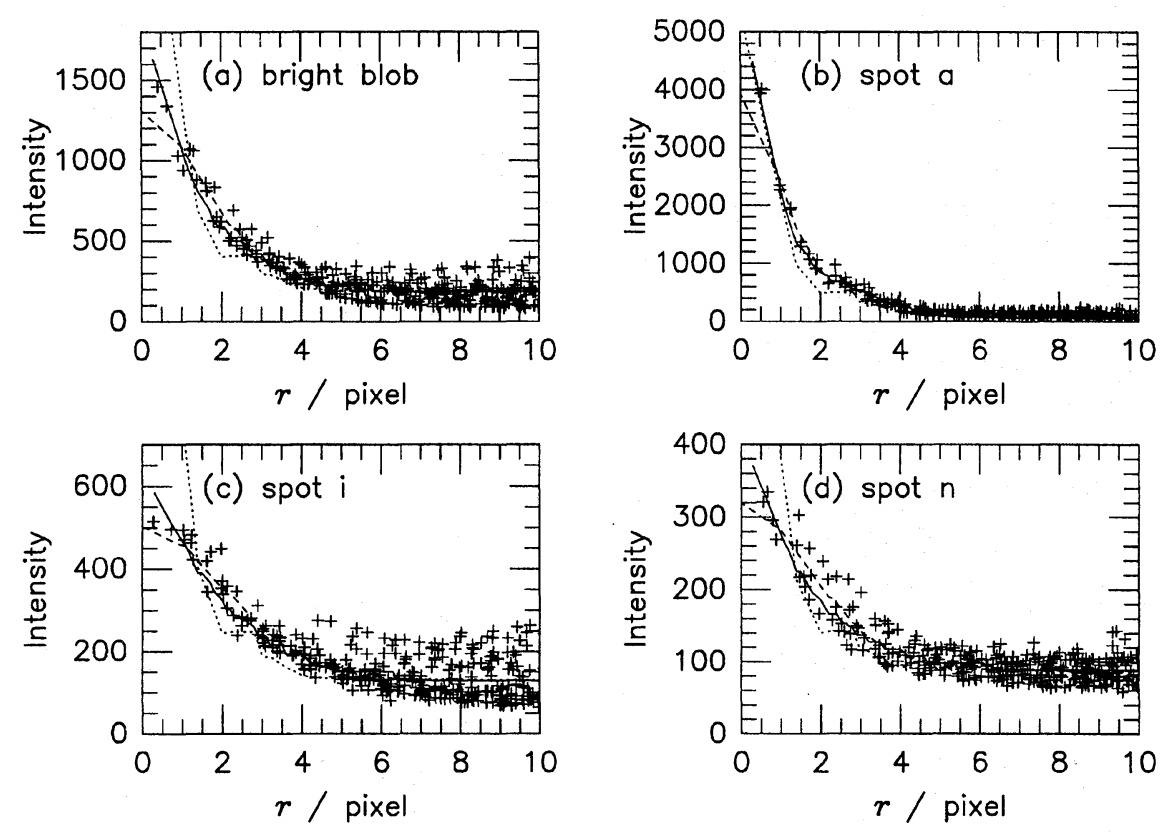

FIG. 6. Radial profiles of the clusters in F814W. The crosses show the data. The dotted line is the PSF. The solid line is the truncated, modified Hubble model and the dashed line is the Gaussian model. Each pixel of 0.045 arcsec or 0.5 parsec.

ing half of the total light). Direct measurement of these from radial profiles requires information at very small radii to determine the peak surface brightness or very large radii to determine the total light. Unfortunately, this information is not available to us because of the finite resolution of HST and because of confusion and low surface brightness beyond radii of a few pixels. We are forced to determine parameters for assumed radial profiles.

We investigated Gaussian and truncated, modified Hubble profiles. Previous authors have often used Gaussian models for compact star clusters. Nevertheless, many resolved star clusters, including old globular clusters and the very young LMC cluster R136 (Hunter et al. 1995) are better fitted by a truncated, modified Hubble profile than a Gaussian profile. A Gaussian profile has $R_{\text {core }}=R_{0.5}=$ HWHM. A truncated, modified Hubble profile has surface brightness proportional to $\left(R_{\text {core }}^{2}+R^{2}\right)^{-1}$ and is truncated at $R=c R_{\text {core }}$. It has $R_{0.5} \approx c^{1 / 2} R_{\text {core }}$ for $c \gg 1$. Away from the core, a modified Hubble profile falls roughly as $R^{-2}$, much less quickly than a Gaussian. The radial profiles (shown in Fig. 6) suggest that $c \gtrsim 10$ for the bright blob, spot $i$, and spot $n$. In the absence of more restrictive constraints we adopted $c=100$, appropriate for many Galactic globular clusters.

To determine the best-fitting parameters for each cluster, we generated five-times over-sampled Gaussian and truncated, modified Hubble models, smoothed them with a fivetimes over-sampled F814W TINYTIM point spread function, and binned them by a factor of five. We then fitted a twodimensional Gaussian plus constant to each model and to the cluster and found the model whose fitted FWHM matched that of the cluster.

The results of this procedure are shown in Fig. 6. The radial profile for each cluster in F814W is shown as crosses, along with the PSF (dotted line) and the appropriate Gauss- ian (dashed line) and truncated, modified Hubble profiles (solid line), after convolution. The best fits are provided by the modified Hubble profiles, although the Gaussian models do well other than in the central few pixels. The difficulty of determining the profiles beyond a few pixels is clear. Spot $n$ shows evidence of elongation.

The derived model parameters are shown in Table 2. With the Gaussian model, the clusters have inferred values of $R_{\text {core }}=R_{0.5}$ between 0.4 and 1.0 parsec and with the truncated, modified Hubble profile the clusters have inferred values of $R_{\text {core }}$ between 0.1 and 0.5 parsec and $R_{0.5}$ between 1 and 5 parsec. Since the half-light radius scales roughly as $c^{1 / 2}$, adopting a value of $c$ closer to 10 would reduce the derived values of $R_{0.5}$ by a factor of about 3 , making them similar to the values for the Gaussian model. The clusters are clearly very compact.

\subsection{Broad Band Photometry}

Photometry within apertures of radii 4 pixels in PC1 centered on the $\mathrm{F} 814 \mathrm{~W}$ centroid of the bright blob gives $m_{555}=21.2$ and $m_{814}=16.6$. We adopted an aperture correction of 1.0 magnitudes for both bands, calculated from the truncated, modified Hubble model for the bright blob, al-

TABLE 2. Cluster sizes.

\begin{tabular}{ccccc}
\hline \hline & Gaussian & \multicolumn{2}{c}{ Modified Hubble } \\
\cline { 3 - 5 } Cluster & $\begin{array}{c}\text { Measured FWHM } \\
\text { pixels }\end{array}$ & $\begin{array}{c}R_{\text {core }} \equiv R_{0.5} \\
\text { parsec }\end{array}$ & $\begin{array}{c}R_{\text {core }} \\
\text { parsec }\end{array}$ & $\begin{array}{c}R_{0.5} \\
\text { parsec }\end{array}$ \\
\hline Bright blob & 4.0 & 0.7 & 0.25 & 2.5 \\
Spot $a$ & 2.9 & 0.4 & 0.10 & 1.0 \\
Spot $i$ & 5.0 & 1.0 & 0.50 & 5.0 \\
Spot $n$ & 4.4 & 0.8 & 0.35 & 3.5 \\
\hline \hline
\end{tabular}


TABLE 3. Cluster photometry.

\begin{tabular}{|c|c|c|c|c|c|c|c|}
\hline Cluster & $m_{555}-m_{814}$ & $m_{814}$ & $J-H$ & $H-K$ & $K$ & $\underset{\operatorname{erg~s}^{-1} \mathrm{~cm}^{-2}}{F_{\mathrm{H} \alpha}}$ & $\underset{\AA}{\mathrm{EW}_{\mathrm{Hc}}}$ \\
\hline Bright blob & 4.6 & 17.6 & 1.06 & 1.13 & 11.44 & $6.8 \times 10^{-15}$ & 250 \\
\hline Spot $a$ & 3.2 & 16.0 & 0.66 & 0.40 & 13.18 & $3.5 \times 10^{-15}$ & 30 \\
\hline Spot $i$ & 3.2 & 16.8 & 1.15 & 0.39 & 13.03 & $5.3 \times 10^{-15}$ & 170 \\
\hline Spot $n$ & 4.4 & 17.9 & 1.28 & 0.50 & 13.00 & $1.0 \times 10^{-15}$ & 160 \\
\hline
\end{tabular}

though this is uncertain to some degree, especially for F555W. Adopting the Gaussian model would make the clusters about 0.8 magnitudes fainter; clearly, the errors are dominated by the uncertainty in the aperture correction arising from our poor knowledge of the profile beyond a few pixels. The color is less uncertain, as the aperture corrections will largely cancel. The bright blob is extremely red with $V-I \approx m_{555}-m_{814}=4.6$. The changes in color within the bright blob suggest that attempts to use this mean color to determine the effective extinction to the bright blob will be hampered by large uncertainties.

We applied the same technique to the other clusters, using aperture corrections based on the truncated, modified Hubble model. The results are shown in Table 3. Again, the errors in the magnitudes are dominated by the aperture corrections, which are similar to those for the bright blob.

Table 3 also lists $J-H, H-K$, and $K$ photometry from Sams et al. (1994) and kindly supplied by Sams. These have errors of about 0.15 in the colors and 0.1 in the magnitudes.

\section{$5.4 \mathrm{H} \alpha$ Flux}

We modeled the observed spectrum of the bright blob near $\mathrm{H} \alpha$ as a continuum $F_{\lambda} \propto \lambda^{\alpha}$ plus line emission. The observed $m_{555}-m_{814}$ color requires $\alpha \approx 8.4$. The observed count rates in 2 pixel radius apertures in $\mathrm{F} 656 \mathrm{~N}$ and $\mathrm{F} 675 \mathrm{~W}$ require $F_{\mathrm{H} \alpha} \approx 6.8 \times 10^{-15} \mathrm{erg} \mathrm{s}^{-1} \mathrm{~cm}^{-2}$ and $\mathrm{EW}_{\mathrm{H} \alpha} \approx 250 \AA$. In our model, we accounted for continuum emission in F656N and $\mathrm{F} 675 \mathrm{~W}$, emission from $\mathrm{H} \alpha$ and $[\mathrm{N} \mathrm{II}] 6548 \AA$ in $\mathrm{F} 656 \mathrm{~N}$, and emission from $\mathrm{H} \alpha$, [N II] $6548 \AA$ and $6584 \AA$, and [S II] $6717 \AA$ and $6731 \AA$ in F675W. We assumed that these lines have the same ratios as average ratio of the inner $\mathrm{H}$ II regions I to VI of M83 (Dufour et al. 1980). [N II] $6584 \AA$, which has been observed to be strong in NGC 253, lies outside F656N.

We applied the same technique to the other clusters and show the results in Table 3.

\subsection{Radio Continuum Flux}

Turner \& Ho (1995) produced a $2 \mathrm{~cm}$ VLA A array image of the central $8 \times 8$ arcsec of NGC 253. Their cleaned beam was $0.2 \times 0.1$ arcsec, comparable to the size of the clusters. The $3 \sigma$ detection limit of their map is $0.4 \mathrm{mJy}$ per beam, so the bright blob must either have a $2 \mathrm{~cm}$ flux density of less than $0.4 \mathrm{mJy}$ or must be identified with one of the other point sources. Sources 7 and 8 are good candidates, as they lie within 0.8 and 0.5 arcsec of our adopted position of the bright blob. These sources have $2 \mathrm{~cm}$ flux densities of 3.3
mJy and brightness temperatures of 1500-3000 K. There are no good candidate counterparts for spots $i$ and $n$; spot $a$ lies beyond their map.

\subsection{Bolometric Luminosity}

A strong point source apparently corresponding to the bright blob is visible in the arcsecond-resolution midinfrared maps of Piña et al. (1992) and Keto et al. (1993). We estimated the bolometric luminosity of the bright blob by scaling the bolometric luminosity in a 15 arcsec radius aperture by the $12.5 \mu \mathrm{m}$ flux density ratio between the bright blob and that aperture. This method has been used by Telesco et al. (1989) in M82.

The peak flux density in the bright blob in the $12.5 \mu \mathrm{m}$ image obtained by Keto et al. (1993) is $1.08 \mathrm{Jy}$. Assuming a Gaussian point spread function with a FWHM of 1.2 arcsec, the total flux density of the bright blob is $1.76 \mathrm{Jy}$ at $12.5 \mu \mathrm{m}$. We choose $12.5 \mu \mathrm{m}$ because it is least contaminated by emission other than dust continuum emission; the $11.3 \mu \mathrm{m}$ dust emission feature lies outside their filter and the spectrum of Roche \& Aitken (1985) suggests that [Ne II] $12.8 \mu \mathrm{m}$ contributes only about $10 \%$. Telesco \& Harper (1980) estimate that the bolometric luminosity of NGC 253 is $1.5 \times 10^{10}$ $L_{\odot}$ (adjusting to a distance of $2.3 \mathrm{Mpc}$ ) within an aperture of radius 15 arcsec. We estimated the $12.5 \mu \mathrm{m}$ flux density in a 15 arcsec radius aperture from the photometry of Rieke \& Low (1975), scaling the small aperture flux density of 11.2 Jy by a factor of 1.75 derived from the small and large aperture ratio of the $10.6 \mu \mathrm{m}$ flux densities. This gives a 12.5 $\mu \mathrm{m}$ flux density of $20 \mathrm{Jy}$.

The bolometric luminosity of the bright blob is therefore about $1.3 \times 10^{9} L_{\odot}$. The uncertainty is probably a factor of 2 . Our value is lower than the value of $3.8 \times 10^{9} L_{\odot}$ derived by Piña et al. (1992), but their images are significantly coarser than the images of Keto et al. (1993) and so are more likely to include additional sources or background. Spot $a$ may be visible as elongations in the contours in the 12 and $20 \mu \mathrm{m}$ images of Piña et al. (1992). Unfortunately, it does not appear in any of the higher resolution maps of Keto et al. (1993), although it might lie just outside their mosaic. No obvious distinct counterparts of spots $i$ and $n$ are visible in any of the mid-infrared images, but they do lie in regions of strong emission.

\section{DERIVED PROPERTIES OF THE CLUSTERS}

We used the work of Leitherer \& Heckman (1995, hereafter LH) to derive further properties of the clusters. These authors modeled the properties of bursts of star formation with different metallicities and three different initial mass functions (IMFs): power-law index 2.35 and masses from $1 M_{\odot}$ to $100 M_{\odot}$ ("Salpeter"); power-law index 3.3 and masses from $1 M_{\odot}$ to $100 M_{\odot}$ ("Miller-Scalo"); and powerlaw index 2.35 and masses from $1 M_{\odot}$ to $30 M_{\odot}$ ("truncated Salpeter'). Given the location of the clusters near the center of a luminous galaxy, we used LH's metal-rich $2 Z_{\odot}$ models.

Of course, those properties of a cluster that are largely derived from massive stars are insensitive to moderately low values of the lower cutoff in the IMF. Changing the lower 

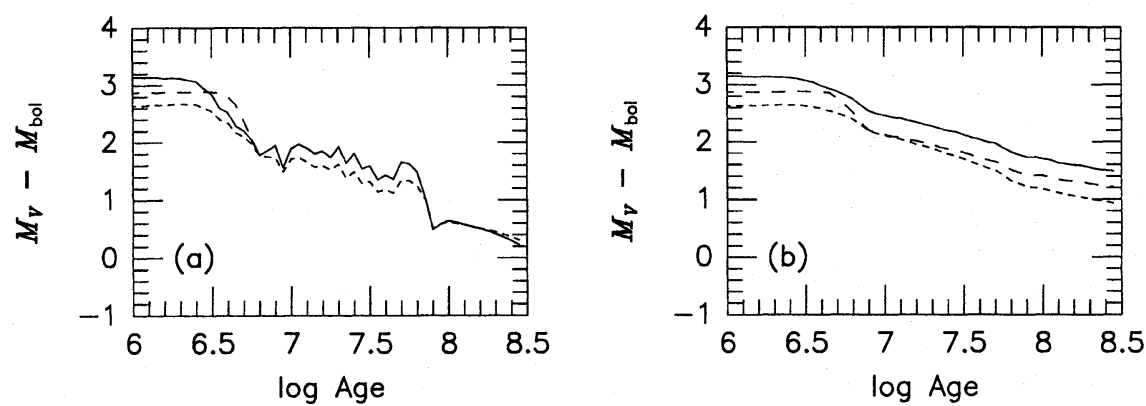

FIG. 7. The evolution of the $M_{V}-M_{\text {bol }}$ color of a cluster from the models of Leitherer \& Heckman (1995). A burst model is shown in (a) on the left and a continuous model in (b) on the right. The three IMFs are denoted by different line styles: solid for the IMF with power-law index 2.35 and masses from $1 M_{\odot}$ to $100 M_{\odot}$ ("Salpeter"); long dashed for the IMF with power-law index 3.3 and masses from $1 M_{\odot}$ to $100 M_{\odot}$ ("Miller-Scalo"); and short dashed for the IMF with power-law index 2.35 and masses from $1 M_{\odot}$ to $30 M_{\odot}$ ("truncated Salpeter").

cutoff of a Salpeter IMF from $1 M_{\odot}$ to $0.08 M_{\odot}, 2.8 M_{\odot}$, or $10 M_{\odot}$ while keeping the mass of massive stars constant changes the total mass by factors of $2.8,0.62$, and 0.31 . The lower cutoff of $2.8 M_{\odot}$ is significant because Hunter et al. (1995) find a relatively normal IMF in the dense LMC super star cluster R136 down at least this far.

\subsection{Robust Limits on $M_{V}$ and the Mass of the Clusters}

If the extinction to the bright blob were uniform, we could estimate the extinction from the $V-I$ color excess. However, the extinction is directly observed to be patchy, with stronger absorption across the central part of the cluster, and so we can only place a rough lower limit on the effective extinction. The bright blob is seen in $\mathrm{H} \alpha$, so it still contains at least some $\mathrm{O}$ stars and can probably be intrinsically no redder than $V-I \approx 1$. The observed color of $V-I \approx 4.6$ then implies $A_{V} \geq 8.5$ and $M_{V} \lesssim-13.3$. Assuming a bluer intrinsic color for the cluster, as it may possibly be, would make this lower limit brighter.

We can place a stronger limit on $M_{V}$ for the bright blob from $M_{\text {bol }}$. Figure 7 shows the predictions of $\mathrm{LH}$ for $M_{V}-M_{\text {bol }}$. Figure 7(a) is for a burst model and Fig. 7(b) for a continuous model. The three IMFs considered by $\mathrm{LH}$ are shown in each plot. (We adjusted the values of $M_{\text {bol }}$ given by LH so that $M_{\mathrm{bol}, \odot}$ has the more conventional value of 4.64.) A robust upper limit on $M_{V}$ for the bright blob is provided by noting that $M_{V}-M_{\text {bol }} \leqslant 3.2$ for all of the $\mathrm{LH}$ models. Thus, the intrinsic $M_{V}$ of the bright blob is brighter than about -14.9 . This limit is shown in Table 4 .

TABLE 4. Cluster derived properties.

\begin{tabular}{|c|c|c|c|c|c|}
\hline Cluster & $M_{\text {bol }}$ & $M_{V}$ & $\begin{array}{c}\operatorname{Mass}^{\mathrm{a}} \\
M_{\odot}\end{array}$ & $L_{\odot, V}^{\Sigma_{0.5}}$ & $\begin{array}{c}\rho_{0.5} \\
M_{\odot} \mathrm{pc}^{-3}\end{array}$ \\
\hline Bright blob & -18.1 & $\leq-14.9^{b}$ & $\gtrsim 1.5 \times 10^{6 b}$ & $\geq 2.0 \times 10^{6}$ & $\gtrsim 1.1 \times 10^{4}$ \\
\hline Spot $a$ & - & $\leq-10.9^{c}$ & $\geq 5 \times 10^{4} \mathrm{c}$ & $\geq 3.2 \times 10^{5}$ & $\gtrsim 6 \times 10^{3}$ \\
\hline Spot $i$ & - & $\leq-11.0^{c}$ & $\geq 6 \times 10^{4} \mathrm{c}$ & $\gtrsim 1.4 \times 10^{4}$ & $\gtrsim 6 \times 10^{1}$ \\
\hline Spot $n$ & - & $\lessgtr-11.1^{\mathrm{c}}$ & $\geq 6 \times 10^{4 \mathrm{c}}$ & $\geq 3.1 \times 10^{4}$ & $\geqq 1.7 \times 10^{2}$ \\
\hline
\end{tabular}

Notes to TABLE 4

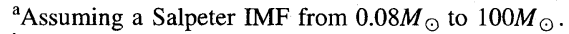

berived from $M_{\text {bol }}$.

${ }^{c}$ Derived from $M_{K}$.
Similarly, we can derive a limit on the mass of the bright blob from a limit on the mass-to-light ratio. Figure 8 shows the predictions of $\mathrm{LH}$ for the bolometric luminosity produced by one million solar masses of stars. (Our normalization for the continuous model is different from LH's; we normalize to one million solar masses at all times whereas they normalize to a star formation rate of one solar mass per year.) The minimum mass-to-light ratio is attained by the Salpeter IMF model at about 2.5 Myr and implies a lower limit to the mass of the bright blob of about $5 \times 10^{5} M_{\odot}$. If we conservatively assume that the IMF extends only to $2.8 M_{\odot}$, we obtain a lower limit for the mass of the bright blob that is in excess of $3 \times 10^{5} M_{\odot}$. If the IMF extends down to $0.08 M_{\odot}$, the lower limit is $1.5 \times 10^{6} M_{\odot}$. This limit is shown in Table 4 .

We do not have a measurement of $M_{\text {bol }}$ for the other clusters, but we can use the observed $K$ magnitude in its place and ignore the effects of extinction (since $V-K \leqq 10$ for the clusters, the extinction at $K$ is likely to be less or about a magnitude). Figure 9 shows the predictions of $\mathrm{LH}$ for the $M_{V}-M_{K}$ color. Since, $M_{V}-M_{K} \leqslant 2.8$, we can place limits on the intrinsic values of $M_{V}$ for the clusters. Figure 10 shows the predictions of LH of $M_{K}$ for one million solar masses of stars. The minimum mass-to-light ratio is reached by the burst models with non-truncated IMFs at about $8 \mathrm{Myr}$. The minimum masses for the clusters are around $5 \times 10^{4}$ solar masses, assuming a Salpeter IMF down to $0.08 M_{\odot}$. These limits are also shown in Table 4.

The bolometric and $K$ mass-to-light ratios rise sharply away from their minimum values. The limits we have derived in this section and listed in Table 4 could easily underestimate the true masses of the clusters by an order of magnitude or more.

\subsection{Further Limits on the Mass and Age of the Bright Blob}

We can constrain the mass and age of the bright blob by considering observed constraints on the ratio of the rate of production of ionizing photons $N_{\text {Lyc }}$ to bolometric luminosity $L_{\text {bol }}$. We shall call $N_{\text {Lyc }} / L_{\text {bol }}$ the "hardness ratio.",

We have two firm limits on the ionizing luminosity. It must be sufficient to provide at least the observed $\mathrm{H} \alpha$ flux but must not produce more than the observed $2 \mathrm{~cm}$ flux density limit of about $3.3 \mathrm{mJy}$. We assume that 

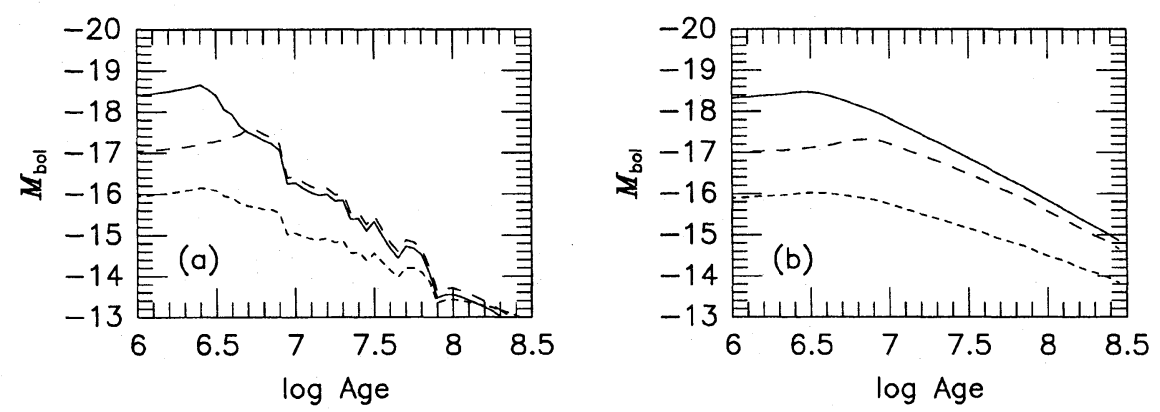

FIG. 8. The evolution of $M_{\text {bol }}$ of a $10^{6} M_{\odot}$ cluster from the models of Leitherer \& Heckman (1995). A burst model is shown in (a) on the left and a continuous model in (b) on the right. The different line styles denote different IMFs (see Fig. 7).

no photons escape from the vicinity of the bright blob. Assuming an optically thin $\mathrm{H}$ II region with a density of $10^{4} \mathrm{~cm}^{-3}$ and a temperature of $5000 \mathrm{~K}$, we find $N_{\text {Lyc }}=1.11 \times 10^{26}\left(L_{2} \mathrm{~cm} / \mathrm{erg} \mathrm{s}^{-1} \mathrm{~Hz}^{-1}\right)$ and $N_{\text {Lyc }}=6.9 \times 10^{11}$ $\times\left(L_{\mathrm{H} \alpha}\right.$ erg s$\left.^{-1}\right)$. Thus, $48.4 \leqq \log N_{\text {Lyc }} \leqslant 51.3$. These limits on $N_{\text {Lyc }}$ allow us to place limits on the hardness ratio $N_{\text {Lyc }} / L_{\text {bol }}$ (which has units of $\mathrm{s}^{-1} L_{\odot}^{-1}$ ) of the radiation emitted by the bright blob: $39.3 \$ \log \left(N_{\text {Lyc }} / L_{\text {bol }}\right) \$ 42.2$. Since $L_{\text {bol }}$ is uncertain by a factor of 2 , we can accept deviations up to about 0.3 outside this interval. It is also of interest to consider the possibility that the bright blob might not be associated with TH 7 or 8 , in which case the upper limit on its $2 \mathrm{~cm}$ flux density is $0.4 \mathrm{mJy}$, so $\log N_{\text {Lyc }} \$ 50.4$ and $\log \left(N_{\text {Lyc }} / L_{\text {bol }}\right)$ $\lesssim 41.3$.

Figure 11(a) shows the change in the hardness ratio $N_{\text {Lyc }} / L_{\text {bol }}$ for an instantaneous burst with the three different IMFs studied by LH. The limits of $\log \left(N_{\mathrm{Lyc}} / L_{\mathrm{bol}}\right)=39.3$, 41.3 , and 42.2 are also shown. It can be seen that the properties of the bright blob are consistent with ages of between about 8 and $50 \mathrm{Myr}$, almost regardless of whether it is associated with TH 7 or 8 . Figure 11(b) shows that mass of stars required to produce the observed bolometric luminosity of the cluster, assuming a lower mass cutoff of $1 M_{\odot}$. The properties of the bright blob are consistent with cluster masses of between $2 \times 10^{6} M_{\odot}$ and about $3 \times 10^{7} M_{\odot}$, consistent with the lower limit we estimated above. These masses increase by a factor of about 2.8 if the IMF extends to $0.08 M_{\odot}$.

Figures $11(\mathrm{c})$ and $11(\mathrm{~d})$ repeat the information in Figs. 11(a) and 11(b) for continuous star formation rate models. If the bright blob is associated with TH 7 or 8 , then its properties are marginally consistent with an age in excess of about $300 \mathrm{Myr}$ and a mass in excess of $2 \times 10^{7} M_{\odot}$, but only if the IMF is non-standard.

\subsection{Limits on the Characteristic Density and Star Formation Intensity of the Clusters}

O'Connell et al. (1994) have used $\Sigma_{0.5}$, the mean surface brightness within the half-light radius in units of $L_{V, \odot} \mathrm{pc}^{-2}$, to characterize the intensity of star formation. This quantity is fairly straightforward to define and has the nice property of being independent of distance, but its interpretation is tricky as it is a strong function of age. From our limits on $M_{V}$ and estimated half-light radii from the truncated, modified Hubble model, the intensity of the bright blob is $\Sigma_{0.5} \geq 2.0 \times 10^{6} L_{V, \odot} \mathrm{pc}^{-2}$. The intensities of the other clusters are listed in Table 4.

We can define a characteristic density $\rho_{0.5}$ for the clusters by dividing half of the mass by the volume within the adopted half-light radius. This gives characteristic densities in excess of $1.1 \times 10^{4} M_{\odot} \mathrm{pc}^{-3}$ for the bright blob, assuming a Salpeter IMF from $0.08 M_{\odot}$ to $100 M_{\odot}$. The characteristic densities of the other clusters are listed in Table 4.

These intensity and characteristic density depend sensitively on the half-light radius, which is uncertain. Adopting a concentration factor $c=10$ instead of $c=100$ or adopting a Gaussian profile would increase the intensity by a factor of about 10 and the density by a factor of about 30 .
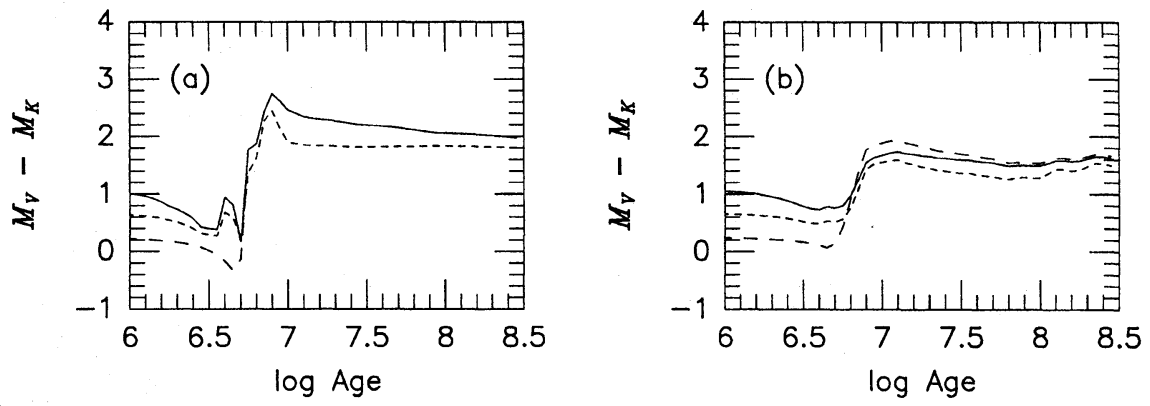

FIG. 9. The evolution of the $M_{V}-M_{K}$ color of a cluster from the models of Leitherer \& Heckman (1995). A burst model is shown in (a) on the left and a continuous model in (b) on the right. The different line styles denote different IMFs (see Fig. 7). 

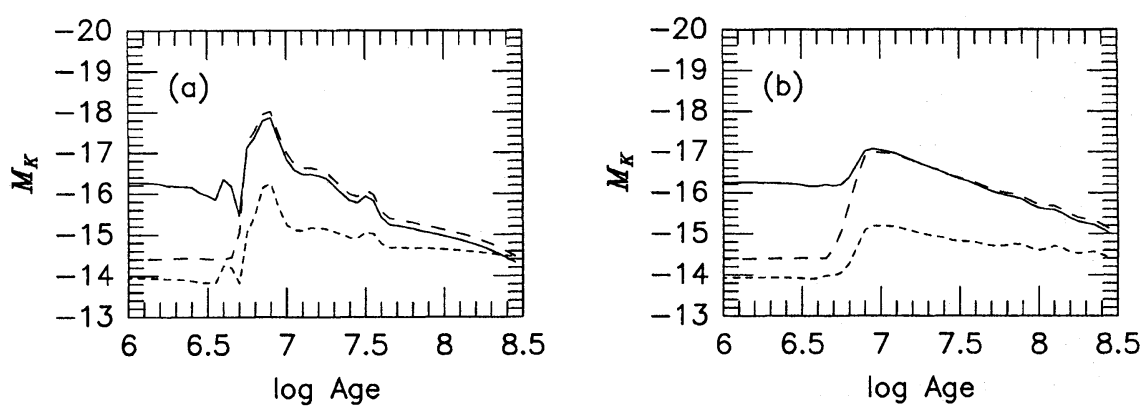

FIG. 10. The evolution of $M_{K}$ of a $10^{6} M_{\odot}$ cluster from the models of Leitherer \& Heckman (1995). A burst model is shown in (a) on the left and a continuous model in (b) on the right. The different line styles denote different IMFs (see Fig. 7).

\section{DISCUSSION}

We have investigated the central region of the starburst spiral galaxy NGC 253 using high resolution visible, nearinfrared, mid-infrared, and radio data. We have found that the bright blob and spots $a, i$, and $n$ are best identified with young, luminous, compact stellar clusters located a few tens of parsec from the nucleus.

The observed properties of the bright blob are consistent with a single age stellar population of less than $50 \mathrm{Myr}$, an intrinsic $M_{V}$ of about -15 or brighter, a core radius $R_{\text {core }}$ of 0.25 parsec, and a mass in excess, possibly well in excess, of $1.5 \times 10^{6} M_{\odot}$, assuming a Salpeter IMF from 0.08 to $100 M_{\odot}$. The half-light radius $R_{0.5}$ depends sensitively on the adopted radial profile. Assuming a profile similar to a galactic globular cluster, a truncated, modified Hubble profile, gives $R_{0.5} \approx 2.5$ parsec, but assuming a more compact profile, a Gaussian or a more severely truncated modified Hubble law gives $R_{0.5} \approx 0.7$ parsec.

The observed properties of spots $a, i$, and $n$ are consistent with an intrinsic $M_{V}$ of about -11 or brighter, core radii of $0.1,0.5$, and 0.35 parsec, and masses in excess of $5 \times 10^{4}$ $M_{\odot}$, again assuming a Salpeter IMF from 0.08 to $100 M_{\odot}$. Again the half-light radii depend sensitively on the assumed profile.

The clusters have masses that are in the range spanned by Milky Way globular clusters, but appear to be somewhat more compact, although it is possible that they will expand as they age.

The bright blob is more luminous than the central cluster in 30 Dor, which has $M_{V} \approx-11.1$; the other clusters are consistent with the luminosity of 30 Dor. All are similar in com-
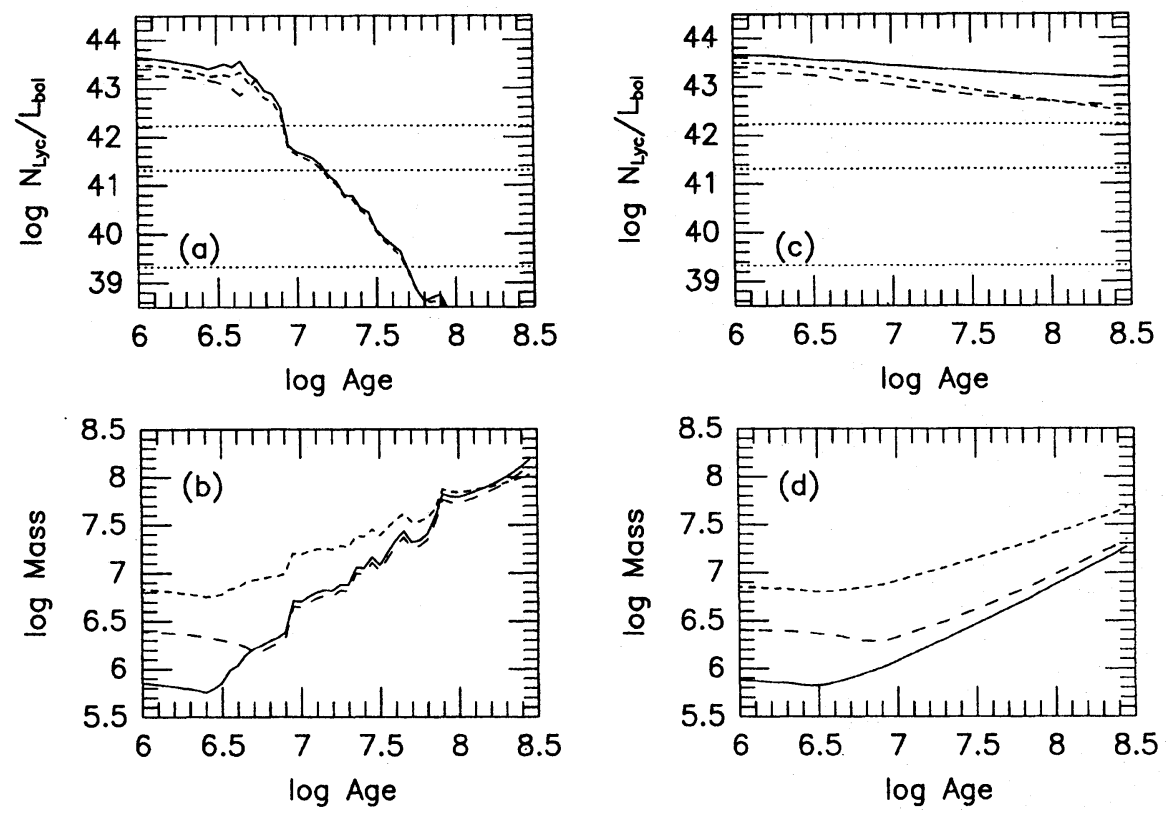

Fig. 11. The evolution of the hardness ratio $N_{\mathrm{Lyc}} / L_{\mathrm{bol}}$ and the required mass of the bright blob. A burst model is shown on the left and a continuous model on the right. The different line styles denote different IMFs (see Fig. 7). The dotted lines are observational limits on the hardness ratio from the observed H $\alpha$ flux ( $\left.\log N_{\mathrm{Lyc}} / L_{\mathrm{bol}} \geq 39.3\right)$ and the $2 \mathrm{~cm}$ radio flux density limits of $0.4 \mathrm{mJy}$ and $3.3 \mathrm{mJy}\left(\log N_{\mathrm{Lyc}} / L_{\mathrm{bol}} \leqslant 41.3\right.$ and 42.2$)$. 
pactness to 30 Dor, which has a half-light radius of 1.7 parsec (Hunter et al. 1995).

The properties of the bright blob are similar to the "super star clusters" and the "candidate young globular clusters" found in other galaxies. The intensity of $\Sigma_{0.5} \lesssim 2.0 \times 10^{6} L_{V, \odot}$ $\mathrm{pc}^{-2}$ is similar to or greater than that of the prototypical super star cluster $A$ in NGC 1569 (O'Connell et al. 1994). It is difficult to compare compactnesses, as most other clusters have had their half-light radii estimated by fitting Gaussians, and this process may give values of $R_{0.5}$ that may be significantly lower then the true values. Nevertheless, it appears that these clusters are similar in compactness to the most compact clusters found elsewhere. The continuing discovery of such clusters in a wide range of host galaxies suggests that they may form wherever star formation is sufficiently intense.

If the bright blob has a composite population and a normal IMF, its properties approach those that might be associated with a galactic nuclear stellar cluster. Since the cluster is located about $40 \mathrm{pc}$ from the nucleus of NGC 253, it would presumably be the nucleus of a galaxy swallowed by NGC 253. The absence of other evidence for a recent merger argues against this possibility.

In other galaxies, the brightest young clusters are often found with less luminous companions, and this seems to be the case in NGC 253 too. It is also possible is that spots $a, i$, and $n$ may be more closely related to the much less massive clusters found in the central regions of the starburst galaxies M83 (Heap et al. 1993) and M100 (Gallagher et al. 1994) than to either of the other classes of more massive clusters.

Zepf \& Ashman (1993) investigated the specific frequency $T$ of old globular clusters per $10^{9} M_{\odot}$ for a number of galaxies and found $T \leqslant 4$ for spirals and $T \leqslant 15$ for ellipticals. If we assume that star formation in the central region has been continuous for the last $10^{9}$ years and has a Salpeter IMF from 0.08 to $100 M_{\odot}$, then the bolometric luminosity of
$1.5 \times 10^{9} L_{\odot}$ corresponds to a mass of about $1.5 \times 10^{8} M_{\odot}$ of young stars (Charlot \& Bruzual 1991; Bruzual \& Charlot 1993). If $T$ for these young stars in only 4 , the probability of finding four young globulars in NGC 253 is only 0.0033 . If $T$ is 15 , it rises to 0.19 .

If we assume that the four clusters in NGC 253 are indeed young globular clusters we are driven to the conclusion that the specific frequency of young globular clusters formed recently in the nuclear region of NGC 253 is significantly higher than the specific frequency of old globular clusters in spiral galaxies. If the starburst is younger than $10^{9}$ years, for example if it is only a few times $10^{7}$ years old, comparable to the expansion time scale of the superwind (McCarthy et al. 1987; Fabbiano 1988), then the specific frequency could be very much higher. This may well be of relevance to the increase in the specific frequency of globular clusters required if ellipticals form from the interactions of spirals (Schweizer 1986; Ashman \& Zepf 1992; Zepf \& Ashman 1993). On the other hand, it may be telling us more about the likelihood that a young globular cluster in a spiral galaxy survives to be observed as an old globular cluster in a spiral galaxy.

The bright blob and spots $a, i$, and $n$ are excellent candidate young globular clusters. However, for them to fulfill their potential, they must survive the ravages of internal and external dynamical forces for a Hubble time and must have IMFs that include low mass stars.

We are grateful to our anonymous referee for comments which significantly improved this paper. We thank Greg Bothun for supplying the wide-field image of NGC 253 from which we derived our astrometric calibration and Bruce Sams for supplying near-infrared photometry. This work made use of the NASA Extragalactic Database and the Digitized Sky Survey. This work was supported by NASA under contract NAS 7-918.

\section{REFERENCES}

Aldering, G. S., \& Bothun, G. D. 1991, PASP, 103, 1296

Ashman, K. M., \& Zepf, S. E. 1992, ApJ, 384, 50

Arp, H., \& Sandage, A. 1985, AJ, 90, 1163

Barth, A. J., Ho, L. C., Filippenko, A. V., \& Sargent, W. L. W. 1995, AJ, 110, 1009

Biretta, J. 1995, private communication

Bruzual, A., G., \& Charlot, S. 1993, ApJ, 405, 538

Burrows, C. J., Ed. 1995, WFPC2 Instrument Handbook, 3rd ed. (STScI, Baltimore)

Charlot, S., \& Bruzual, A., G. 1991, ApJ, 367, 126

Conti, P. S., \& Vacca, W. D. 1994, ApJ, 423, L97

Combes, F., Gottesman, S. T., \& Weliachew, L. 1977, A\&A, 59, 181

Cox, C. 1994, private communication

Crabtree, D. R., \& Smecker-Hane, T. A. 1994, BAAS, 26, 1499

Davidge, T. J., \& Pritchett, C. J. 1990, AJ, 100, 102

Davidge, T. J., Le Fèvre, O., \& Clark, C. C. 1991, ApJ, 370, 559

Dufour, R. J., Talbot, R. J., Jensen, E. B., \& Shields, G. A. 1980, ApJ, 236, 119

Fabbiano, G. 1988, ApJ, 330, 672

Gallagher, J. S., et al. 1994, BAAS, 26, 939

Groth, E. 1991, in WF/PC Final Orbital/Science Verification Report, edited by S. Faber (STScI, Baltimore), Chap. 14
Heap, S. R., Holbrook, J., Malumuth, E., Shore, S., \& Waller, W. 1993, BAAS, 25, \#31.04

Holtzman, J. A. 1995, in preparation

Holtzman, J. A., Burrows, C. J., Watson, A. M., \& Worthy, G. S. 1995b, PASP, 107, 1065

Holtzman, J. A., et al. 1992, AJ, 103, 691

Holtzman, J. A., et al. 1995a, PASP, 107, 156

Hunter, D. A., O'Connell, R. W., \& Gallagher, J. S. 1994, AJ, 108, 84

Hunter, D. A., Shaya, E. J., Holtzman, J. A., Light, R. M., O’Neil, Jr., E. J., \& Lynds, R. 1995, ApJ, 448, 179

Keto, E., Ball, R., Arens, J., Jernighan, G., Meixner, M., Skinner, C., \& Graham, J. 1993, ApJ, 413, L23

McCarthy, P. J., Heckman, T. M., \& van Breugel, W. 1987, AJ, 93, 264

Melnick, J., Moles, M., \& Terlevich, R. 1985, A\&A, 149, L24

Meurer, G. R., Freeman, K. C., Dopita, M. A., \& Cacciari, C. 1992, AJ, 103, 60

Meurer, G. R., Heckman, T. M., Leiterer, C., Kinney, A., \& Robert, C. 1995, AJ, 110, 2665

Leitherer, C., \& Heckman, T. M. 1995, ApJS, 96, 9

Lutz, D. 1991, A\&A, 245, 31

O'Connell, R. W., Gallagher, J. S., \& Hunter, D. A. 1994, ApJ, 433, 65

O'Connell, R. W., Gallagher, J. S., Hunter, D. A., \& Colley, W. N. 1995, ApJ, 446, L1 
Piña, R. K., Jones, B., Puetter, R. C., \& Stein, W. A. 1992, ApJ, 401, L75

Puche, D., Carignan, C., \& van Gorkom, J. H. 1991, AJ, 101, 456

Rieke, G. H., \& Low, F. J. 1975, ApJ 197, 17

Roche, P. F., \& Aitken, D. K. 1985, MNRAS, 213, 789

If Russell, J. L., Lasker, B. M., McLean, B. J., Sturch, C. R., \& Jenkner, H. III 1990, AJ, 99, 2059

Russell, J. L., \& Williams, C. A. 1986, in Astrometric Techniques, IAU Symposium No. 109, edited by H. K. Eichhorn and R. J. Leacock (Reidel, Dordrecht), p. 259

Sams, B. J., Genzel, R., Eckart, A., Tacconi-Garman, L., \& Hofmann, R. 1994, ApJ, 430, L33

Schweizer, F. 1986, in Nearly Normal Galaxies, edited by S. Faber (Springer, New York), p. 18

Schweizer, F., \& Seitzer, P. 1993, ApJ, 417, L29
Telesco, C. M., Decher, R., \& Joy, M. 1989, ApJ, 343, L13

Telesco, C. M., \& Harper, D. A. 1980, ApJ, 235, 392

Trauger, J. T., et al. 1994, ApJ, 435, L3

Turner, J. L., \& Ho, P. T. P. 1985, ApJ, 299, L77

Waller, W. H. 1991, ApJ, 370, 144

Watson, A. M. 1994, Ph.D. thesis, University of Wisconsin-Madison

Watson, A. M., \& Gallagher, J. S. 1996, AJ (submitted)

Whitmore, B. C., \& Schweizer, F. 1995, AJ, 109, 960

Whitmore, B. C., Schweizer, F., Leitherer, C., Borne, K., \& Robert, C. 1993, AJ, 106, 1354

Witt, A. N., Thronson, H. A., \& Capuano, J. M. 1992, ApJ, 393, 611

Zepf, S. E., \& Ashman, K. M. 1993, MNRAS, 264, 611

Zepf, S. E., Carter, D., Sharples, R. M., \& Ashman, K. 1995, ApJ, 445, L19 


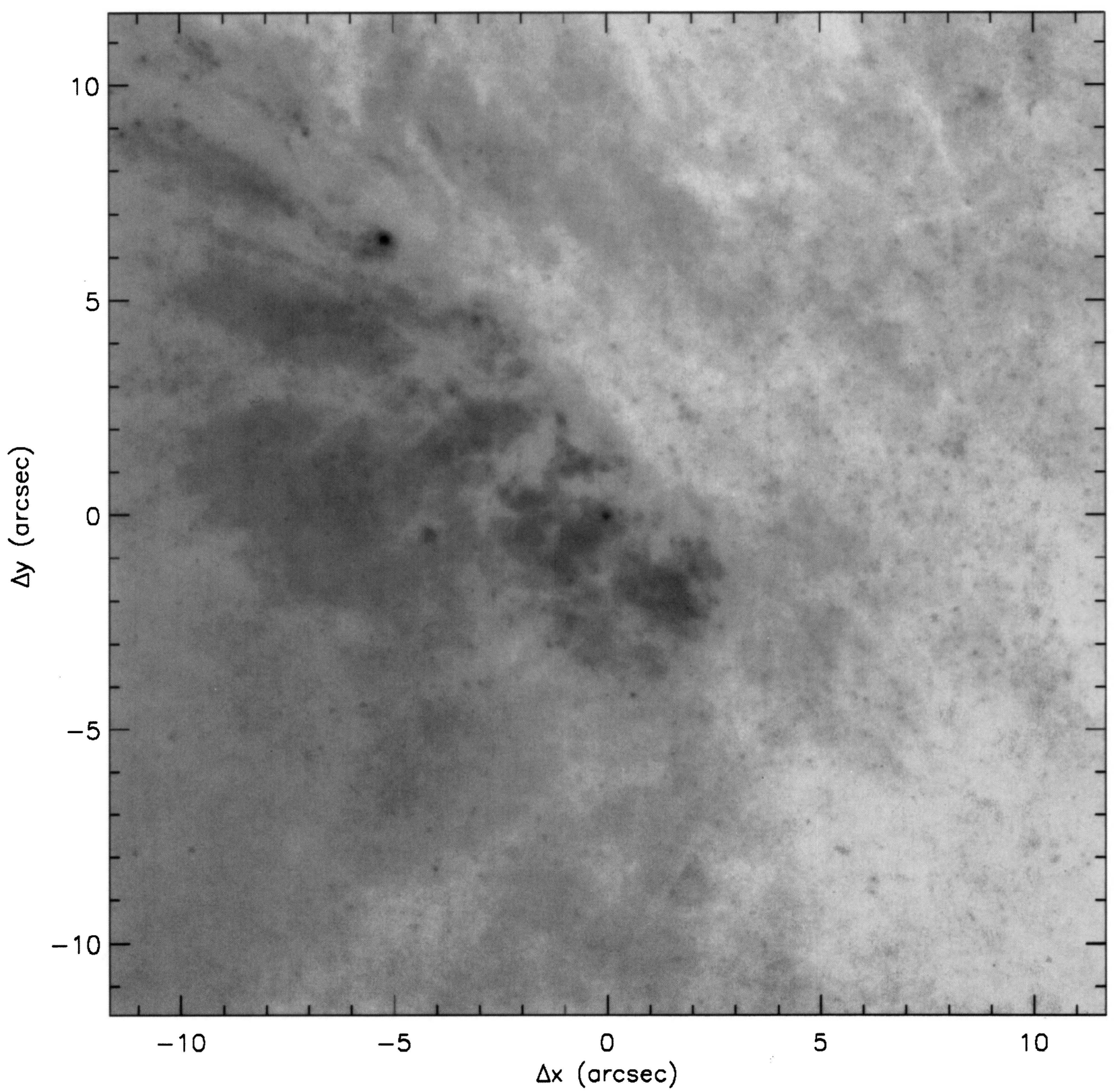

FIG. 1. A F814W image of the nuclear region of NGC 253. The intensity scale is negative and logarithmic over 7.5 magnitudes. The image is about 23 arcsec to a side. North is 7.8 degrees clockwise from vertical and east is roughly to the left. The bright blob is located at the center $(\Delta x=\Delta y=0)$.

Watson et al. (see page 536) 


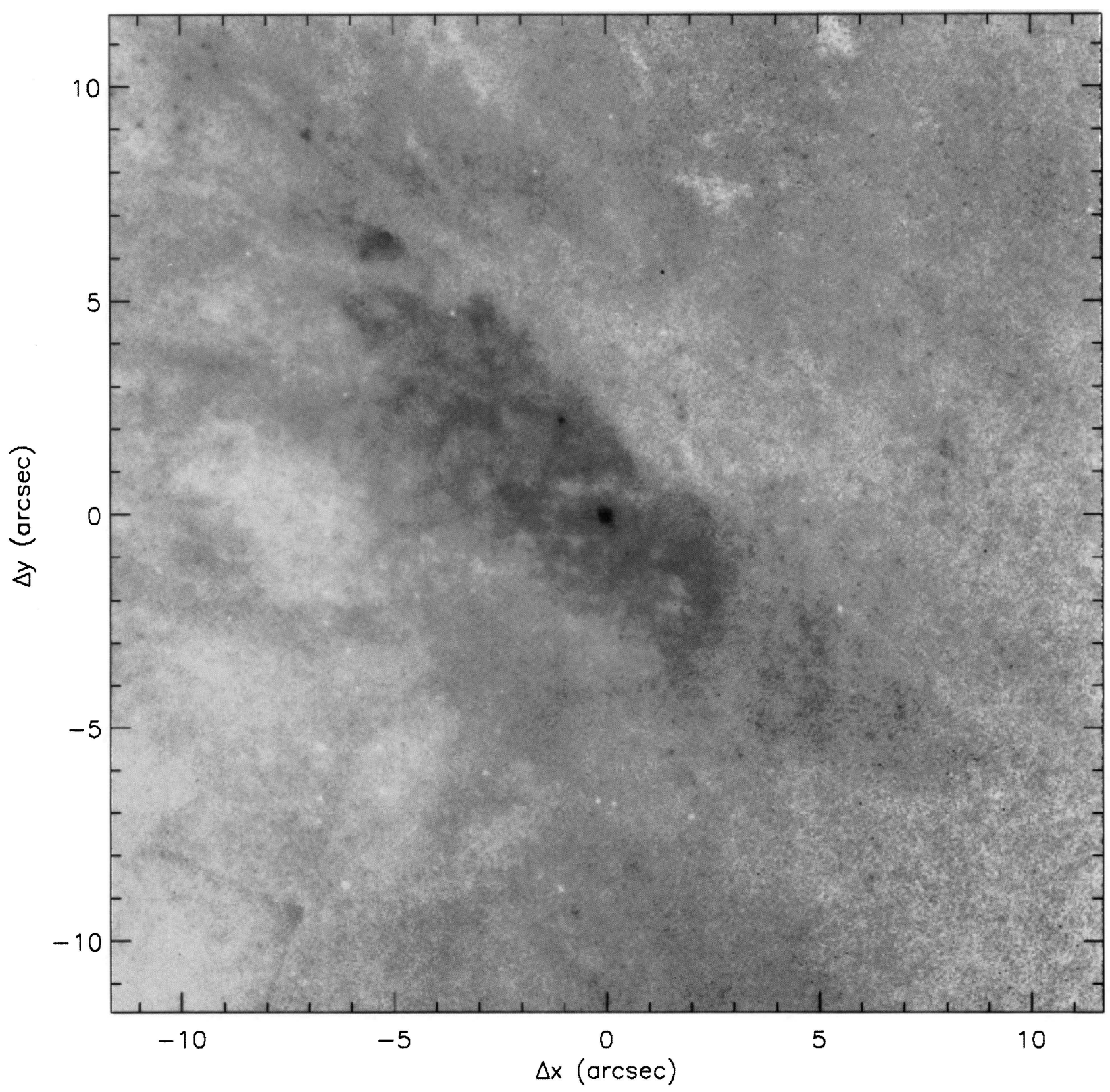

FIG. 2. A $m_{555}-m_{814}$ image of the nuclear region of NGC 253. The scale and orientation are the same as Fig. 1. The intensity scale is linear in magnitudes from $V-I \approx m_{555}-m_{814}=1$ to $V-I \approx m_{555}-m_{814}=5$. 


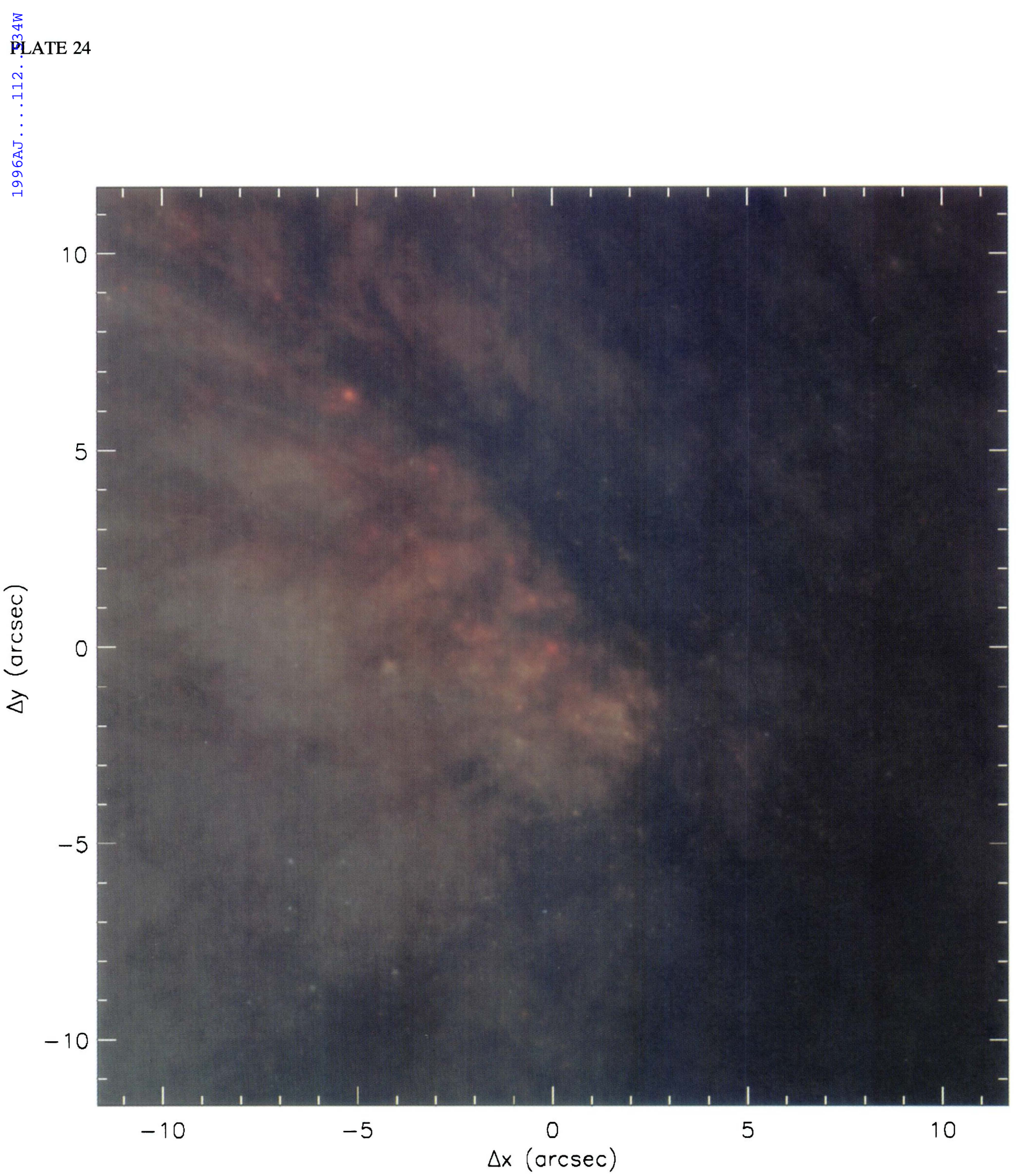

FIG. 3. A color image of the nuclear region of NGC 253 constructed from the F555W and F814W images. The scale and orientation are the same as Fig. 1. The intensity scale is positive and roughly logarithmic and the color scale is linear.

Watson et al. (see page 536) 


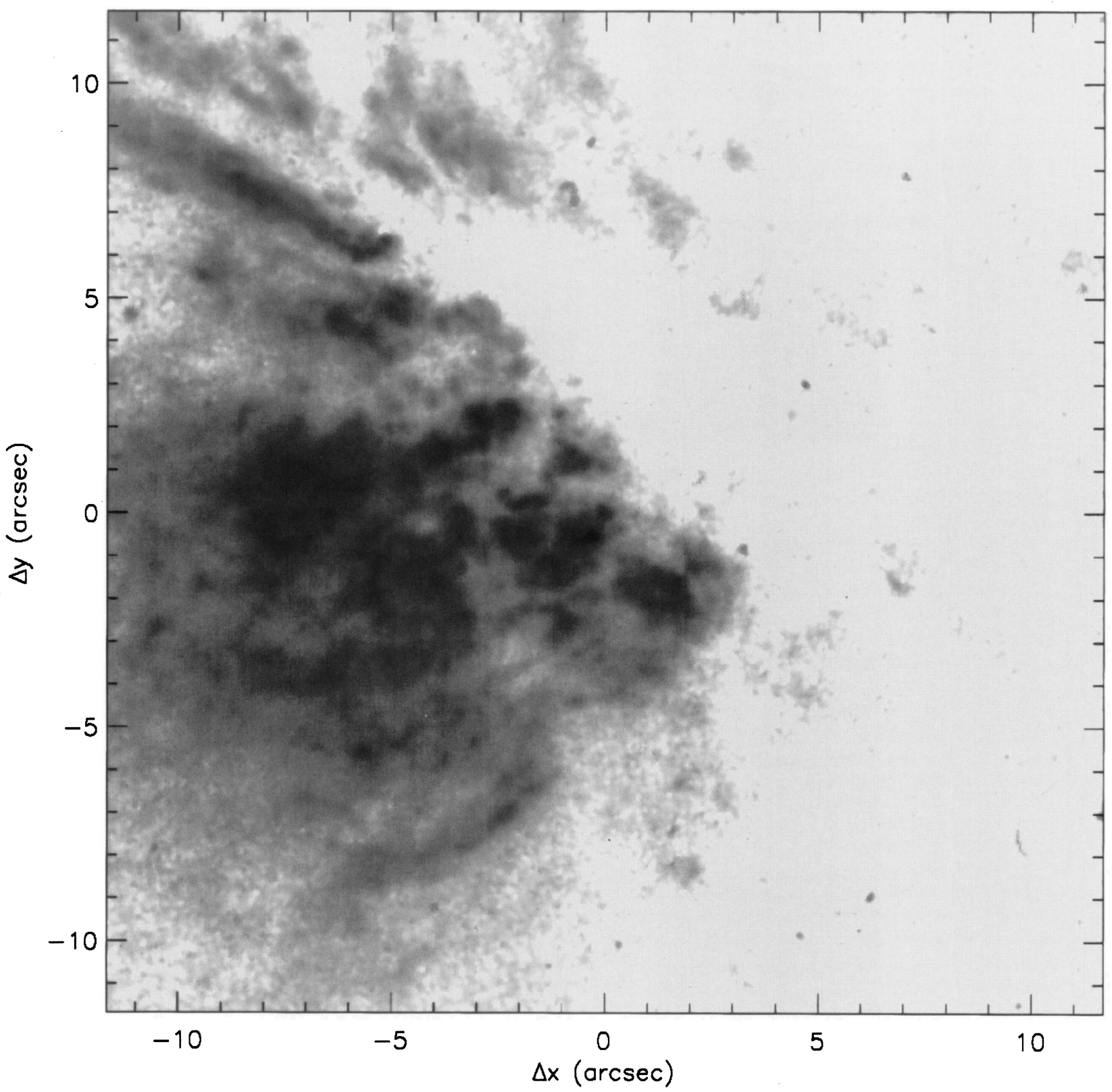

FIG. 4. A continuum subtracted $\mathrm{H} \alpha$ image constructed from our WF3 images of the nuclear region in F656N and F675W. The image has been rotated and oversampled to have the same scale and orientation as Fig. 1, but has intrinsically lower resolution. The intensity scale is negative and logarithmic over 10 magnitudes.

Watson et al. (see page 536) 
染

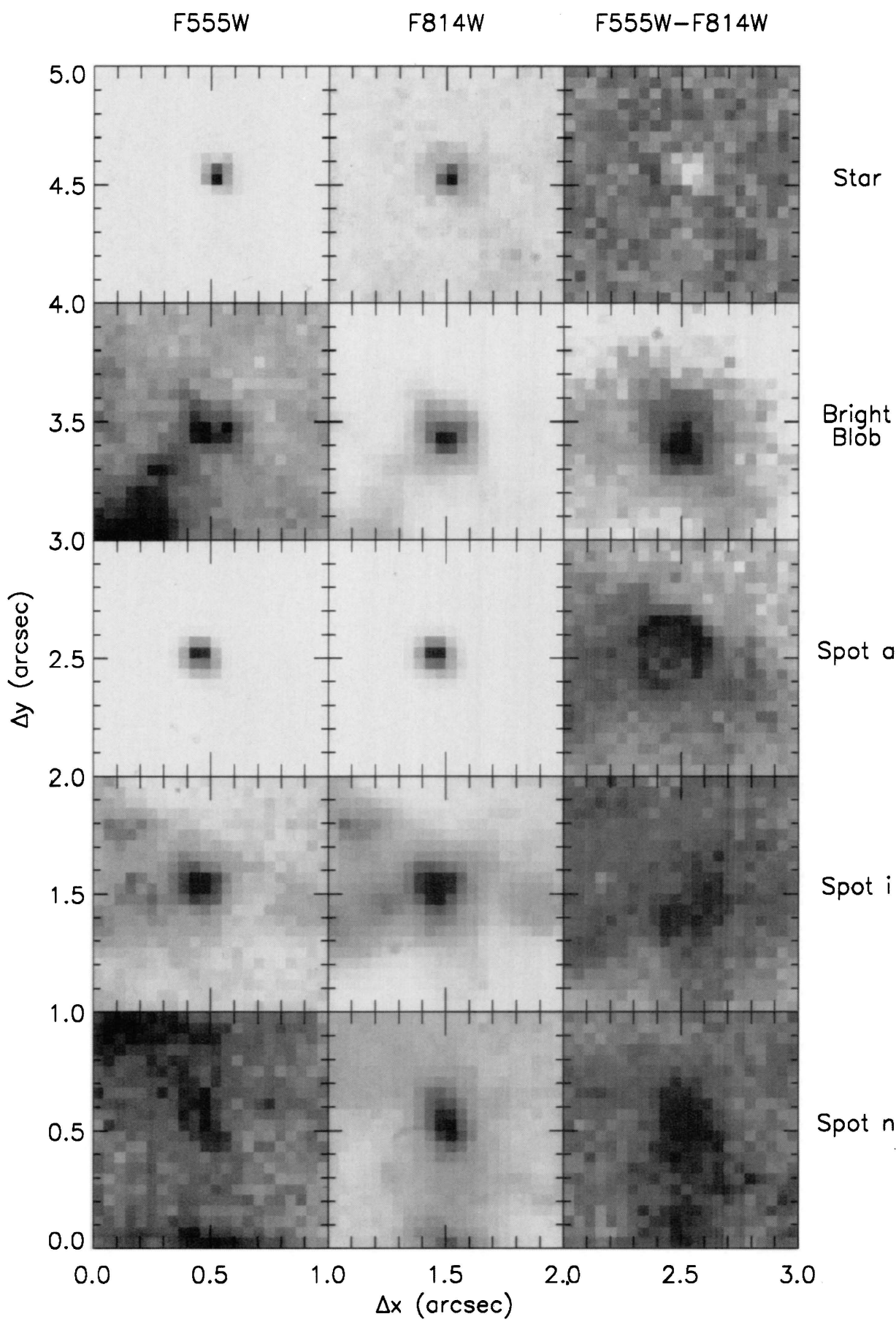

FIG. 5. The clusters and a star in the F555W (left), F814W (center), and $m_{555}-m_{814}$ PC1 images. The F555W and F814W images have individual stretches that are linear in counts and the $m_{555}-m_{814}$ images have individual stretches that span 2.5 magnitudes. Each image is 22 PC1 pixels or 1.0 arcsec to a side.

Watson et al. (see page 536) 